\title{
El estado de la investigación en educación en cirugía general en Colombia (2000-2020): un análisis bibliométrico
}

\author{
The state of research in general surgery education in Colombia \\ (2000-2020): A bibliometric analysis \\ José Manuel Sánchez-Jaramillo ${ }^{1} \mathbb{D}$, Luis Carlos Domínguez ${ }^{2} \mathbb{D}$, Neil Valentín Vega $^{3} \mathbb{D}$, \\ Paula Meneses ${ }^{4} \mathbb{D}$

\begin{abstract}
Médico, especialista en Cirugía General, Universidad de la Sabana, Chía, Colombia. Médico, especialista en Cirugía General; profesor, Departamento de Cirugía, Universidad de la Sabana, Chía, Colombia. Médico, especialista en Cirugía General; director, Departamento de Cirugía, Universidad de la Sabana, Chía, Colombia.

Medica, especialista en Cirugía General; Universidad de la Sabana, Chía, Colombia.
\end{abstract}

\section{Resumen}

Introducción. Se desconoce el estado de la investigación en educación médica en cirugía general (IEMC) en Colombia en el siglo XXI. El objetivo de esta revisión bibliométrica de la literatura es realizar un análisis de las publicaciones relacionadas con la IEMC en Colombia a partir del año 2000.

Métodos. Se incluyeron artículos originales, escritos en español o inglés, publicados por grupos de investigación que cuentan al menos con un investigador colombiano como autor principal o coautor. Se definieron variables demográficas e indicadores bibliométricos para cada estudio y autor.

Resultados. Un total de 63 estudios fueron incluidos. Estas publicaciones se enfocaron predominantemente en investigación sobre bienestar, enseñanza clínica y simulación a nivel de postgrado. El 36 \% de los artículos sobre IEMC fueron publicados en revistas sin indexación (ISI/SCOPUS); 13 artículos (20,6\%) fueron publicados en revistas en el cuartil 1 (Q1). El promedio de citas por artículo fue 9,3.

Discusión. La producción intelectual en educación en cirugía en Colombia tiene bajo impacto a nivel internacional. Los hallazgos encontrados pueden ser utilizados para organizar y priorizar la investigación en educación quirúrgica en el país.

Palabras clave: educación médica; cirugía general; programas de postgrado; investigación; impacto; factor de impacto; bibliometría.

Fecha de recibido: 17/06/2020 - Fecha de aceptación: 30/08/2020 - Fecha de publicación en línea: 24/02/2021

Correspondencia: José Manuel Sánchez-Jaramillo, Campus del Puente del Común, Km. 7, Autopista Norte de Bogotá. Chía, Colombia. Teléfono: 8615555 / 8616666 / 3132103512. Correo electrónico: manolojmsj@gmail.com

Citar como: Sánchez-Jaramillo JM, Domínguez LC, Vega NV, Meneses P. El estado de la investigación en educación en cirugía general en Colombia (2000-2020): un análisis bibliométrico. Rev Colomb Cir. 2021;36:205-20. https://doi.org/10.30944/20117582.702

Este es un artículo de acceso abierto bajo una Licencia Creative Commons - BY-NC-ND https://creativecommons.org/licenses/by-ncnd/4.0/deed.es 


\begin{abstract}
Introduction. The state of research in medical education in general surgery (IEMC) in Colombia in the XXI century is unknown. The objective of this bibliometric review of the literature is to conduct an analysis of the publications related to the IEMC in Colombia from the year 2000.
\end{abstract}

Methods. Original articles, written in Spanish or English, published by research groups that have at least one Colombian researcher as main author or co-author, were included. Demographic variables and bibliometric indicators were defined for each study and author.

Results. A total of 63 studies were included. Publications predominantly focused on wellness research, clinical teaching, and simulation at the graduate level; $36 \%$ of the articles on IEMC were published in non-indexed journals (ISI / SCOPUS), 13 articles (20.6\%) were published in journals in quartile 1 (Q1). The average number of citations per article was 9.3.

Discussion. Intellectual production in Colombian surgery education shows low impact at international level. These findings can be used to prioritize research in surgical education in the country.

Keywords: medical education; general surgery; health postgraduate programs; research; impact; impact factor; bibliometrics.

\section{Introducción}

La investigación en educación médica en cirugía general (IEMC) es necesaria para mejorar la calidad de la educación y el cuidado quirúrgico ${ }^{1-3}$.

En consonancia con lo anterior, es evidente un aumento en el volumen de publicaciones científicas, facilitado por la incorporación de recursos tecnológicos (como simulación, aula invertida o e-learning), la integración disciplinar (bioingeniería) y la tecnología biomédica en educación quirúrgica, los cuales han sido acogidos por gran parte de la comunidad académica ${ }^{4-7}$

El desarrollo y el perfeccionamiento de habilidades, técnicas y no técnicas, en los cirujanos es influenciado por la IEMC, con el fin de mejorar los desenlaces clínicos y la seguridad del paciente, obteniendo mayor utilidad cuando se implementa en estudiantes de postgrado y en programas de educación médica continuada ${ }^{8,9}$. La IEMC también ha incorporado aspectos relacionados con el bienestar de estudiantes, residentes y cirujanos, en el contexto en el cual se surte su actividad educativa y profesional ${ }^{10}$. De igual manera, han aumentado las publicaciones relacionadas con la calidad de vida del cirujano, la influencia del género, las disparidades y la cirugía global, entre otros 7,10,11.

En Colombia es limitada la documentación de la actividad académica de los cirujanos, así como su experiencia educativa en la práctica diaria, siendo evidente la poca disponibilidad de estudios relacionados con su efectividad, cumplimiento e impacto ${ }^{12-14}$. Una forma de establecer el desempeño de esta actividad es mediante un análisis metódico de los productos derivados de su implementación en escenarios académicos. La cienciometría, ciencia que estudia y analiza la producción científica mediante métodos cualitativos, cuantitativos (análisis bibliométrico) y computacionales ${ }^{15}$, se constituye en un elemento evaluativo útil, que utiliza los indicadores bibliométricos como instrumentos de evaluación ${ }^{16,17}$.

Por otra parte, la evaluación mediante los indicadores bibliométricos es aplicable a la IEMC, permitiendo establecer tendencias, vacíos, posibles focos de intervención e investigación colaborativa multicéntrica, para así facilitar la consolidación de la actividad educativa y académica de los cirujanos, como agentes de cambio transformadores de su entorno.

Estudios realizados en Canadá, Estados Unidos y Brasil ${ }^{18-20}$, han analizado el número de citas obtenidas por artículos sobre educación en cirugía en revistas indexadas, pero carecen de análisis bibliométricos rigurosos ${ }^{21}$. A nivel latinoamericano, a partir del año 2000, la IEMC ha tenido un desarrollo sostenido en países como Argentina, 
Chile, Colombia y México ${ }^{22-24}$, pero su estado, caracterización metodológica, áreas de cobertura y desarrollos temáticos secuenciales en líneas de investigación, no están documentados de forma amplia.

Los autores de este estudio consideramos el vacío de conocimiento como una oportunidad para determinar el estado actual de la IEMC en Colombia y, de acuerdo con esto, identificar áreas de trabajo colaborativo, articulación interinstitucional y desarrollo de nuevas líneas de investigación, en el contexto nacional y latinoamericano. El presente trabajo busca responder la pregunta: ¿Cuál es el estado actual de la IEMC en Colombia (2000-2020), desde una perspectiva bibliométrica?

\section{Metodo \\ Instrumentos}

Se utilizó una matriz en Excel, donde se realizó el registro documental basado en el protocolo PRIS$\mathrm{MA}^{25}$, recomendado para el desarrollo de revisiones bibliográficas, sistemáticas y meta-análisis, en donde se registraron autores, título del artículo, año de la publicación, idioma, indexación de la revista, objetivo, metodología, análisis realizados e instrumentos de medida.

\section{Diseño del estudio y procedimiento}

Este estudio es de tipo descriptivo, basado el análisis bibliométrico y la revisión sistemática de los documentos seleccionados. Para responder la pregunta de investigación realizamos una revisión bibliométrica (RB) de la literatura con el fin de identificar, evaluar, interpretar y sintetizar todas las investigaciones en el campo de la IEMC realizadas por autores colombianos desde enero del año 2000 hasta junio del 2020. No se consideraron autores extranjeros dado el foco del estudio relacionado con la IEMC efectuada en un contexto local y producto de la actividad médica y educativa de los autores colombianos.

Inicialmente revisamos las bases de datos MEDLINE, PubMed, EMBASE (Excerpta Medica Data Base), Cochrane, ERIC (Education Resources Information Center), Publindex, BIREME (Bibliote- ca Regional de Medicina, denominada actualmente Centro Latinoamericano y del Caribe de Información en Ciencias de la Salud), SCOPUS, SciELO (Scientific Electronic Library Online) y Google Scholar. Adicionalmente buscamos otros estudios mediante referencias cruzadas. Utilizamos las siguientes palabras clave en idioma español: "cirugía general”, "educación”, “Colombia”, "pregrado", "postgrado", "educación continuada", "simulación", "evaluación", "currículo", "e-learning”, "bienestar", "educación médica", "aprendizaje”, "enseñanza”, "entrenamiento", “TICs", "profesionalismo", "supervisión”, "autonomía”, "residentes", "estudiantes”. Las palabras clave en inglés fueron: "General surgery", "education”, “Colombia”, "medical students", "undergraduates", "residents", "continuous professional development", "simulation", "assessment", "curriculum", "e-learning", "wellbeing”, "learning", "teaching", "Training", "supervision", "professionalism", "autonomy", "residents", "medical students". Los resultados fueron probados con todos los años correspondientes.

En MEDLINE se realizó una búsqueda genérica para recuperar la mayor cantidad de publicaciones, utilizando los términos clave. En SCOPUS se realizó la búsqueda en títulos/ resúmenes/ palabras clave, con el fin de mejorar los resultados. En Google Scholar se utilizaron las palabras claves mencionadas y se realizó la búsqueda de manera genérica. En SciELO se utilizaron palabras claves y uso de filtros en búsqueda avanzada en relación con los criterios previos. Con apoyo del software VOSviewer ${ }^{26}$ se elaboró una red con los principales ejes de búsqueda asociados a las palabras claves de las publicaciones (figura 1)

Incluimos estudios originales publicados en revistas indexadas en idioma inglés o español (estudios transversales, longitudinales, experimentales, revisiones sistemáticas de la literatura y reportes originales de experiencias educativas) sobre IEMC realizados a nivel de pregrado, postgrado o educación continuada, que fueron publicados por grupos de investigación, que cuentan al menos un investigador colombiano como autor principal o coautor (cirujanos o no cirujanos). Excluimos publicaciones no originales (cartas, editoriales, reflexiones y resúmenes de congresos, artículos 


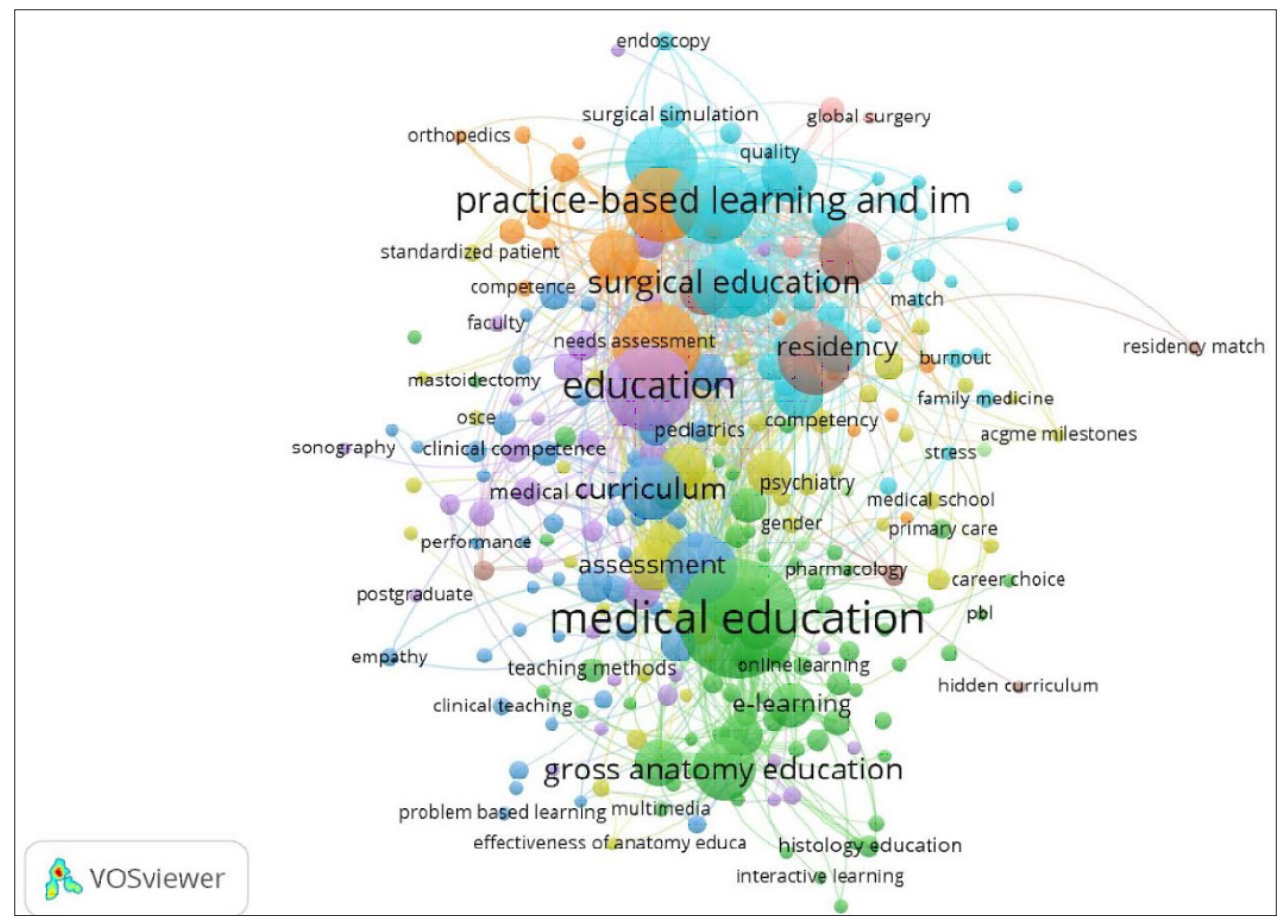

Figura 1. Principales ejes de búsqueda.

de posición, revisiones y publicaciones relacionadas en otras áreas diferentes a la cirugía general). También se excluyeron estudios diseñados para evaluar estrategias de educación para el paciente, efectividad de intervenciones quirúrgicas y aspectos del ejercicio profesional y de fuerza laboral no educativos, así como estudios sobre IEMC en Colombia realizados por autores no colombianos.

De acuerdo con estos criterios y estrategias de búsqueda, los investigadores revisaron de manera independiente la selección preliminar de los estudios. Posteriormente, revisaron los resúmenes de cada estudio y, en conjunto, llegaron a un acuerdo sobre las publicaciones a incluir, teniendo en cuenta los criterios mencionados.

La información obtenida se registró y analizó en programa Excel ${ }^{\circledR}$ (Microsoft Corp), obteniendo frecuencias, medias, rangos y porcentajes.

\section{Variables y desenlaces analizados}

Inicialmente, identificamos las variables demográficas de los estudios (autor, año de publicación, idioma, revista, tipo de universidad en donde fue realizado el estudio y objetivos del estudio). Así mismo, las variables relacionadas con la metodología (diseño, nivel de educación y campo de investigación en educación médica).

En la revisión bibliométrica definimos los siguientes cuatro indicadores de impacto, con base en indicadores bibliométricos comúnmente utilizados en Web of Science y Thompson Reuters ${ }^{16,27,28}$.

A. Indicadores de impacto relacionados con el artículo científico:

- Indicador de tendencia: número de publicaciones en relación con el tiempo de observación del estudio (2000-2020) ${ }^{16}$.

- Indicador de influencia o reconocimiento:

a. Citaciones en Google Scholar: número de citaciones que ha tenido el artículo, tanto en documentos indexados como no indexados ${ }^{28}$. La fecha de corte para evaluar el número de citaciones fue el 1 de junio de 2020. 
b. Índice $\mathrm{H}$ de la revista: relación entre la calidad de los artículos publicados en la revista (en función del número de citas recibidas de cada artículo) y la cantidad de artículos publicados. Para calcular este índice se organizan todos los artículos de la revista según el número de citas recibidas en orden descendente y se numeran. El índice $\mathrm{H}$ se obtiene en el momento en que el número del orden coincide con el número de citas recibidas por dicho artículo. Por ejemplo: un índice $\mathrm{H}$ de 6 significa que hay 6 artículos que han recibido al menos 6 citas cada uno ${ }^{16}$.

- Indicador de eficiencia: Se determinó por el Factor de impacto (SJR) de la revista en el año de publicación del artículo, el cual evalúa la influencia científica de la revista mediante el número de citas de cada artículo publicado y la importancia o prestigio de las revistas de donde provienen estas $\operatorname{citas}^{16}$. El indicador de eficiencia se evaluó por el último reporte del factor de impacto reportado por las revistas incluidas en el SJR en junio de 2020.

- Indicador de evaluación comparativa: Se utilizó la clasificación por cuartiles (ISI/SCOPUS), la cual representa la posición, según el factor de impacto, de una revista con relación a todas las de su área, siendo el cuartil 1 (Q1) la que representa mejor posición ${ }^{16}$. Este indicador se presenta de acuerdo con el cuartil de cada revista en el año de publicación del artículo.

B. Indicadores de impacto relacionados con los autores de los artículos

Adicionalmente, identificamos los cinco autores colombianos con más publicaciones en esta revisión bibliométrica y evaluamos los siguientes tres indicadores de impacto:

- Indicador de productividad:

a. Número de publicaciones totales.

b. Número de publicaciones en educación médica.
- Indicador de influencia:

a. Índice H (Google Scholar): Se calcula de la misma forma que el índice $\mathrm{H}$ de la revista, pero teniendo en cuenta las publicaciones del autor ${ }^{16}$. La fecha de corte para evaluar el índice $\mathrm{H}$ de cada autor fue el 1 de junio de 2020.

b. Número de citaciones en Google Scholar: Número de citaciones, en documentos indexados como no indexados, que ha tenido el autor según el buscador Google Scholar ${ }^{28}$. La fecha de corte para evaluar el número de citaciones de cada autor en Google Scholar fue el 1 de junio de 2020.

c. Índice H (SCOPUS): Se calcula de la misma forma que el índice $\mathrm{H}$ de la revista, pero teniendo en cuenta las publicaciones del autor, según SCOPUS. La fecha de corte para evaluar el índice $\mathrm{H}$ de cada autor fue el 1 de junio de 2020

- Razón de publicaciones en educación médica por autor con respecto al número de publicaciones generales por autor.

\section{Resultados}

Se identificaron un total de 248 artículos. En la primera revisión se excluyeron 115 artículos sobre áreas de educación diferentes a cirugía. Posteriormente fueron excluidas 56 publicaciones por su diseño metodológico (cartas editoriales o artículos de reflexión) y otras 14 diseñadas para evaluar estrategias de educación en pacientes. Finalmente 63 publicaciones cumplieron criterios de inclusión de esta RB. En la figura 2 se observa el proceso de identificación y selección de los estudios de acuerdo con las recomendaciones universales ${ }^{25}$.

\section{Características demográficas de los artículos}

Un total de veinte publicaciones (31,7\%), fueron escritas en inglés. Cuarenta y siete publicaciones $(74,6 \%)$ fueron realizadas en universidades o 


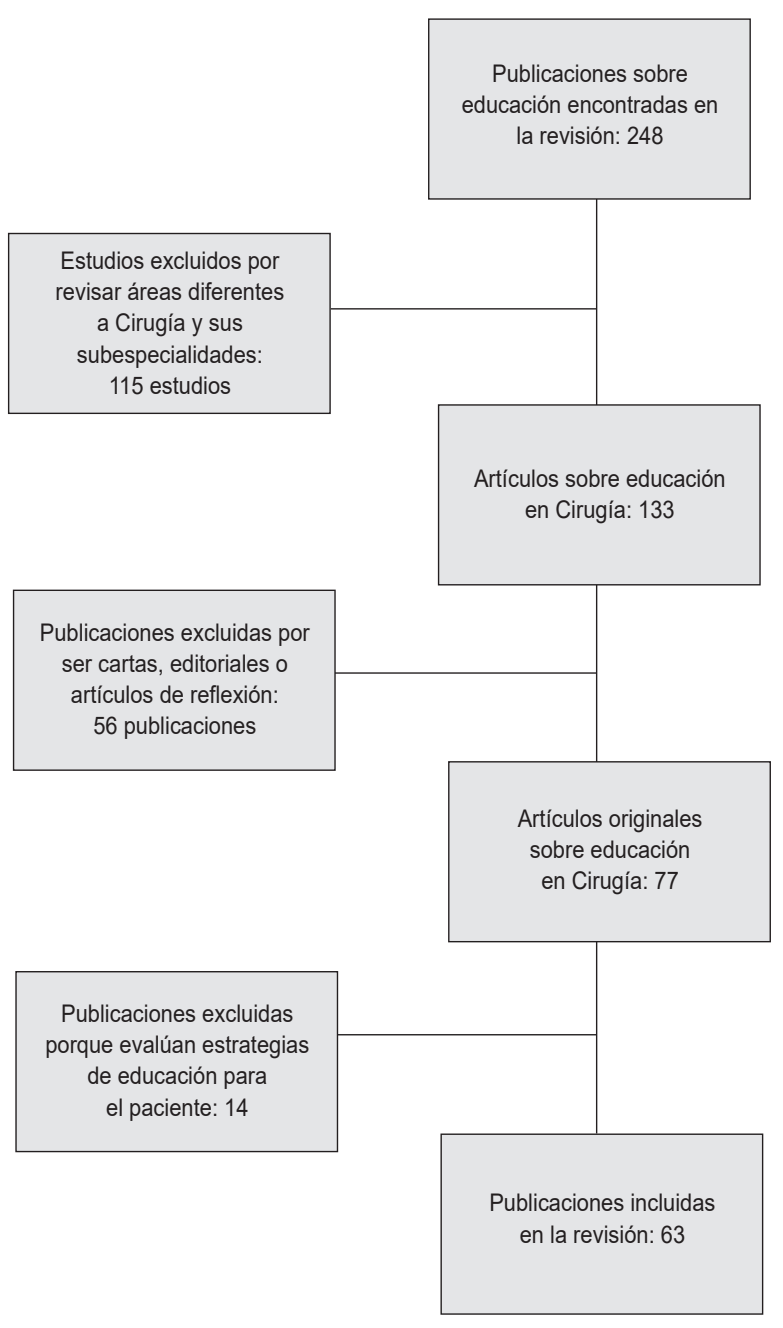

Figura 2. Descripción de la revisión sistemática de las publicaciones.

instituciones privadas. Un total de veintiocho publicaciones $(44,4 \%)$ fueron diseñados a nivel de postgrado, veintitrés a nivel de pregrado (36,5\%) y doce (19\%) en educación continuada (tabla 1).

De acuerdo con el diseño, veinticinco artículos fueron de tipo transversal $(39,7 \%)$, ocho de diseño pretest - postest $(12,5 \%)$ y cinco se desarrollaron para la validación de instrumentos $(7,9 \%)$. Los veinticinco artículos restantes $(40,6 \%)$ se distribuyeron de manera heterogénea entre otros diseños.

Según el campo de IEMC, un total de once estudios $(17,4 \%)$ fueron desarrollados para evaluar
Tabla 1. Características demográficas de los estudios publicados

\begin{tabular}{lc}
\hline \multicolumn{1}{c}{ Característica } & $\begin{array}{c}\text { Numero de estudios } \\
\text { (porcentaje) }\end{array}$ \\
\hline Idioma & $43(68,3 \%)$ \\
Español & $20(31,7 \%)$ \\
Inglés & \\
Nivel Educativo & $23(36,5 \%)$ \\
Pregrado & $28(44,4 \%)$ \\
Postgrado & $12(19,1 \%)$ \\
Educación Continuada & $12(19,1 \%)$ \\
Procedencia de los autores según universidad \\
Pública & $47(74,6 \%)$ \\
Privada & $4(6,3 \%)$ \\
Procedencia mixta &
\end{tabular}

el bienestar estudiantil (en pregrado o postgrado), nueve $(14,3 \%)$ la enseñanza clínica, once $(17,2 \%)$ estrategias pedagógicas, ocho $(12,7 \%)$ simulación y otros ocho (12,7\%) e-learning. Sólo una publicación $(1,2 \%)$ fue diseñada para evaluar el desarrollo profesional continuo (tabla 2).

Treinta y un artículos (49,2\%) se publicaron en revistas nacionales (Revista Colombiana de Cirugía, Revista Ciencias de la Salud, Biomédica, Iatreia y Universitas Medica) y 32 (50,8 \%) en revistas internacionales (Educación médica, Medical Education, Journal of Surgical Research, BMC Medical Education y PloS ONE).

\section{Impacto e influencia de los estudios en IEMC}

A. Indicadores de impacto relacionados con el artículo científico.

- Indicadores de tendencia y producción

En cuanto a la producción anual, se identificó una tendencia creciente en la producción intelectual publicada en revistas científicas a partir de 2000 (figura 3), con el mayor número de publicaciones en 2017 y 2018. En promedio se publicaron 3,14 $\pm 2,94$ artículos /año. 
- Indicadores de influencia

El artículo con mayor número de citaciones en Google Scholar (59 citaciones) fue "La simulación clínica y el aprendizaje virtual. Tecnologías complementarias para la educación médica", realizado por Ruiz-Parra \& colaboradores ${ }^{29}$ publicado en la revista de la Facultad de Medicina de la Universidad Nacional de Colombia (2009). En promedio, el número de citaciones fue de 9,3 , pero quince publicaciones $(23,8 \%)$ no han sido citadas a 2020 (tabla 3).
- Indicadores de eficiencia

Cuarenta artículos (63,5\%) fueron publicados en revistas con clasificación ISI/SCOPUS, con índice $\mathrm{H}$ que varía entre 0 - 268; 14 publicaciones se encuentran en el Q3 y las revistas en donde fueron publicados tienen un índice SJR que oscilan entre 0,101 y 2,085 (Studies Health Technology Informatics, Biomédica, Colombia Médica, Educación médica, Revista Colombiana de Anestesiología) (tabla 2 y figura 4).

Tabla 2. Indicadores demográficos de los artículos incluidos en la revisión.

\begin{tabular}{|c|c|c|c|c|c|c|}
\hline Referencia & Idioma & $\begin{array}{c}\text { Nivel de } \\
\text { educación }\end{array}$ & Diseño & Revista & $\begin{array}{c}\text { Campo de } \\
\text { investigación médica }\end{array}$ & $\begin{array}{c}\text { Tipo de } \\
\text { universidad }\end{array}$ \\
\hline Sanabria et al. 200240 & Español & $\begin{array}{l}\text { Educación } \\
\text { continuada }\end{array}$ & Transversal & $\begin{array}{c}\text { Revista Colombiana } \\
\text { de Cirugía }\end{array}$ & Enseñanza clínica & Pública \\
\hline Riveros et al. $2005^{41}$ & Inglés & Pregrado & Transversal & Stud Health Technol Inform & $\begin{array}{c}\text { Estrategias pedagógicas } \\
\text { (e-learning) }\end{array}$ & Privada \\
\hline Villaveces et al. $2005^{42}$ & Inglés & Pregrado & Transversal & Injury Prevention & Bienestar estudiantil & Pública \\
\hline Bermúdez et al. $2006^{43}$ & Español & Postgrado & Transversal & $\begin{array}{c}\text { Revista Colombiana } \\
\text { de Cirugía }\end{array}$ & Bienestar estudiantil & Privada \\
\hline Isaza et al. $2006^{44}$ & Español & Pregrado & Transversal & $\begin{array}{c}\text { Revista ciencias de } \\
\text { la salud }\end{array}$ & $\begin{array}{c}\text { Aprendizaje asistido por } \\
\text { pares (Peer assisted learning) }\end{array}$ & Privada \\
\hline Isaza et al.2006 ${ }^{45}$ & Español & Pregrado & Transversal & $\begin{array}{l}\text { Revista ciencias de } \\
\text { la salud }\end{array}$ & Bienestar estudiantil & Privada \\
\hline Torregrosa et al. $2009^{46}$ & Español & Postgrado & Transversal & $\begin{array}{c}\text { Revista Colombiana } \\
\text { de Cirugía }\end{array}$ & Enseñanza clínica & Privada \\
\hline Parra-Ruiz et al. $2009^{29}$ & Español & $\begin{array}{l}\text { Educación } \\
\text { continuada }\end{array}$ & Revisión narrativa & $\begin{array}{l}\text { Revista de la facultad } \\
\text { de Medicina }\end{array}$ & $\begin{array}{l}\text { Estrategias pedagógicas } \\
\text { (simulación) }\end{array}$ & Pública \\
\hline Salinas et al. $2009^{37}$ & Español & Pregrado & Diseño de prototipo & Revista chilena de ingeniería & $\begin{array}{c}\text { Estrategias pedagógicas } \\
\text { (simulación) }\end{array}$ & Pública \\
\hline Bejarano et al. $2009{ }^{47}$ & Español & $\begin{array}{l}\text { Educación } \\
\text { continuada }\end{array}$ & Transversal & $\begin{array}{c}\text { Revista Colombiana } \\
\text { de Cirugía }\end{array}$ & Investigación en educación & Pública \\
\hline Tapias et al. 201048 & Español & Postgrado & Transversal & $\begin{array}{c}\text { Revista Colombiana } \\
\text { de Cirugía }\end{array}$ & Bienestar estudiantil & Pública \\
\hline Rubiano et al. $2010^{49}$ & Inglés & $\begin{array}{l}\text { Educación } \\
\text { continuada }\end{array}$ & Pretest - postest & Prehospital Emergency Care & Estrategias pedagógicas & Pública \\
\hline Rojas et al. $2011^{50}$ & Inglés & Postgrado & $\begin{array}{l}\text { Validación de } \\
\text { instrumentos }\end{array}$ & Stud Health Technol Inform & $\begin{array}{l}\text { Estrategias pedagógicas } \\
\text { (simulación) }\end{array}$ & Privada \\
\hline Hull et al. $2012^{51}$ & Inglés & Postgrado & Pretest - postest & $\begin{array}{c}\text { International Journal } \\
\text { of Surgery }\end{array}$ & Estrategias pedagógicas & Pública \\
\hline Henao et al. $2013^{52}$ & Español & $\begin{array}{l}\text { Educación } \\
\text { continuada }\end{array}$ & Pretest - postest & Biomédica & $\begin{array}{c}\text { Estrategias pedagógicas } \\
\text { (simulación) }\end{array}$ & Mixta \\
\hline Domínguez et al. $2013^{53}$ & Español & Postgrado & Transversal & $\begin{array}{c}\text { Revista Colombiana } \\
\text { de Cirugía }\end{array}$ & Desarrollo profesional continuo & Privada \\
\hline Sanabria et al. $2013^{54}$ & Español & $\begin{array}{l}\text { Educación } \\
\text { continuada }\end{array}$ & Revisión narrativa & $\begin{array}{c}\text { Revista Colombiana } \\
\text { de Cirugía }\end{array}$ & Enseñanza clínica & Privada \\
\hline Amaya et al. $2013^{55}$ & Español & $\begin{array}{l}\text { Educación } \\
\text { continuada }\end{array}$ & $\begin{array}{c}\text { Estudio secundario } \\
\text { de revisión de la } \\
\text { literatura }\end{array}$ & $\begin{array}{c}\text { Revista Colombiana de } \\
\text { Cirugía }\end{array}$ & $\begin{array}{l}\text { Aprendizaje asistido por pares } \\
\text { (Peer assisted learning) }\end{array}$ & Pública \\
\hline Ramírez et al. $2014{ }^{56}$ & Español & Postgrado & Pretest - postest & Revista ciencias de la salud & $\begin{array}{c}\text { Estrategias pedagógicas } \\
\text { (simulación) }\end{array}$ & Privada \\
\hline Bustamante et al. $2014{ }^{57}$ & Español & Pregrado & $\begin{array}{l}\text { Validación de } \\
\text { instrumentos }\end{array}$ & Biomédica & Profesionalismo & Mixta \\
\hline Bustamante et al. $2014{ }^{58}$ & Español & Pregrado & Transversal & $\begin{array}{c}\text { Revista Colombiana de } \\
\text { Cirugía }\end{array}$ & Profesionalismo & Mixta \\
\hline Amaya et al. $2014{ }^{59}$ & Inglés & $\begin{array}{l}\text { Educación } \\
\text { continuada }\end{array}$ & $\begin{array}{l}\text { Validación de } \\
\text { instrumentos }\end{array}$ & $\begin{array}{c}\text { International Journal of } \\
\text { Surgery }\end{array}$ & Evaluación estudiantil & Pública \\
\hline Olmos-Vega et al. $2015^{62}$ & Inglés & Postgrado & Métodos mixtos & BMC Medical Education & Enseñanza clínica & Privada \\
\hline Domínguez et al. $2015^{39}$ & Español & Pregrado & Transversal & Biomédica & $\begin{array}{c}\text { Estrategias pedagógicas } \\
\text { (e-learning) }\end{array}$ & Privada \\
\hline
\end{tabular}


Continuación tabla 2

\begin{tabular}{|c|c|c|c|c|c|c|}
\hline Referencia & Idioma & $\begin{array}{c}\text { Nivel de } \\
\text { educación }\end{array}$ & Diseño & Revista & $\begin{array}{c}\text { Campo de investigación } \\
\text { médica }\end{array}$ & $\begin{array}{c}\text { Tipo de } \\
\text { universidad }\end{array}$ \\
\hline Domínguez et al. $2015^{63}$ & Inglés & Pregrado & Pretest - postest & Medical Education & $\begin{array}{l}\text { Aprendizaje asistido por pares } \\
\text { (Peer assisted learning) }\end{array}$ & Privada \\
\hline Barrera et al. $2016^{64}$ & Español & Postgrado & Experimental & Revista Ciencias de la Salud & $\begin{array}{c}\text { Estrategias pedagógicas } \\
\text { (simulación) }\end{array}$ & Privada \\
\hline González-Neira et al. $2016{ }^{65}$ & Inglés & Postgrado & Experimental & Colombia Medica & Evaluación estudiantil & Privada \\
\hline Isaza et al. $2016^{34}$ & Español & Pregrado & Transversal & Revista Ciencias de la Salud & Bienestar estudiantil & Privada \\
\hline Quintero et al. $2016{ }^{66}$ & Inglés & Pregrado & Revisión narrativa & $\begin{array}{l}\text { Journal of Medical Education } \\
\text { and Curricular Development }\end{array}$ & Estrategias Pedagógicas & Privada \\
\hline Bayter et al. $2017{ }^{67}$ & Español & Postgrado & Transversal & $\begin{array}{c}\text { Repertorio de medicina y } \\
\text { cirugía. }\end{array}$ & Enseñanza clínica & Privada \\
\hline Olmos-Vega et al. $2017{ }^{68}$ & Inglés & Postgrado & Cualitativo & Medical Education & Enseñanza clínica & Privada \\
\hline Cervera et al. $2017{ }^{69}$ & Español & Postgrado & Longitudinal & $\begin{array}{c}\text { Investigación en Educación } \\
\text { Médica }\end{array}$ & $\begin{array}{c}\text { Estrategia pedagógica } \\
\text { (e-learning) }\end{array}$ & Privada \\
\hline Domínguez et al. $2017^{70}$ & Español & Postgrado & Transversal & $\begin{array}{c}\text { Revista Colombiana de } \\
\text { Cirugía }\end{array}$ & Bienestar estudiantil & Mixta \\
\hline Domínguez et al. $2017^{71}$ & Español & Pregrado & Pretest - postest & $\begin{array}{l}\text { Revista Colombiana de } \\
\text { anestesiología }\end{array}$ & $\begin{array}{l}\text { Estrategias pedagógicas } \\
\text { (simulación - e-learning) }\end{array}$ & Privada \\
\hline Domínguez et al. $2017^{72}$ & Español & Pregrado & Correlación & Educación Medica & $\begin{array}{c}\text { Estrategias pedagógicas } \\
\text { (simulación) }\end{array}$ & Privada \\
\hline Domínguez et al. $2017^{73}$ & Español & Postgrado & Longitudinal & Educación Medica & $\begin{array}{l}\text { Evaluación programática / } \\
\text { evaluación estudiantil }\end{array}$ & Privada \\
\hline Ramírez et al. $2017^{35}$ & Español & Postgrado & $\begin{array}{l}\text { Revisión de la } \\
\text { literatura }\end{array}$ & latreia & $\begin{array}{l}\text { Estrategia pedagógica } \\
\text { (e-learning) }\end{array}$ & Privada \\
\hline Herrera-Almario et al. $2017^{36}$ & Inglés & Postgrado & Correlación & $\begin{array}{c}\text { The American Journal } \\
\text { of Surgery }\end{array}$ & Evaluación estudiantil & Privada \\
\hline Domínguez et al. $2018^{74}$ & Español & Pregrado & $\begin{array}{l}\text { Validación de } \\
\text { instrumentos }\end{array}$ & Educación Médica & Evaluación estudiantil & Privada \\
\hline Domínguez et al. $2018^{75}$ & Español & Pregrado & Longitudinal & Revista Chilena de Cirugía & Evaluación programática & Privada \\
\hline Domínguez et al. $2018^{76}$ & Español & Postgrado & $\begin{array}{c}\text { Revisión sistemática } \\
\text { de la literatura }\end{array}$ & Educación Medica & Enseñanza clínica & Privada \\
\hline Domínguez et al. $2018^{77}$ & Inglés & Postgrado & $\begin{array}{l}\text { Transversal- } \\
\text { cuantitativo }\end{array}$ & PloS ONE & Bienestar estudiantil & Privada \\
\hline Vergel et al. $2018^{78}$ & Inglés & Pregrado & $\begin{array}{l}\text { Experiencia } \\
\text { institucional }\end{array}$ & Medical education Online & Bienestar estudiantil & Privada \\
\hline Domínguez et al. $2018^{79}$ & Español & Pregrado & Pretest - Postest & Educación Medica & Estrategias pedagógicas & Privada \\
\hline Sierra et al. $2018^{80}$ & Español & Postgrado & Transversa & $\begin{array}{l}\text { Revista Colombiana de } \\
\text { Cirugía }\end{array}$ & Estrategias pedagógicas & Privada \\
\hline Domínguez et al. $2018^{81}$ & Español & Pregrado & Métodos mixtos & Educación Medica & Bienestar estudiantil & Privada \\
\hline Moros et al. $2018^{82}$ & Español & Pregrado & Transversal & Revista Chilena de Cirugía & Estrategias pedagógicas & Privada \\
\hline Castañeda et al. $2019^{83}$ & Español & Postgrado & Revisión narrativa & latreia & Estrategias pedagógicas & Privada \\
\hline Dominguez et al. $2019^{84}$ & Inglés & Postgrado & $\begin{array}{l}\text { Validación de } \\
\text { Instrumentos }\end{array}$ & $\begin{array}{l}\text { International Journal } \\
\text { of Medical Education }\end{array}$ & Bienestar estudiantil & Privada \\
\hline Cortés et al. $2019^{85}$ & Español & Postgrado & Transversal & Universitas Medica & Enseñanza clínica & Privada \\
\hline Dominguez et al. $2019^{86}$ & Inglés & Posgrado & Cualitativo & Journal of Surgical Research & Bienestar estudiantil & Privada \\
\hline Dominguez et al. $2019^{87}$ & Español & Pregrado & Transversal & Biomédica & Estrategias pedagógicas & Privada \\
\hline Angarita et al. $2019{ }^{88}$ & Inglés & Pregrado & Transversal & $\begin{array}{l}\text { Breast Cancer Research } \\
\text { and Treatment }\end{array}$ & Enseñanza clínica & Privada \\
\hline Orlas et al. $2020^{89}$ & Inglés & Pregrado & Transversal & Journal of Surgical Research & Enseñanza clínica & Privada \\
\hline Dominguez et al. $2020{ }^{90}$ & Español & Pregrado & Transversal & Revista Chilena de Cirugía & Profesionalismo & Privada \\
\hline Cabrera et al. $2020^{91}$ & Español & Postgrado & Revisión narrativa & $\begin{array}{l}\text { Revista Colombiana de } \\
\text { Cirugía }\end{array}$ & Estrategias pedagógicas & Privada \\
\hline Sánchez et al. $2020^{38}$ & Español & Postgrado & Revisión narrativa & $\begin{array}{l}\text { Revista Colombiana de } \\
\text { Cirugía }\end{array}$ & Estrategias pedagógicas & Privada \\
\hline Cabrera et al. $2020^{92}$ & Inglés & $\begin{array}{l}\text { Educación } \\
\text { continuada }\end{array}$ & Transversal & Surgery & $\begin{array}{c}\text { Estrategias pedagógicas } \\
\text { (e-learning) }\end{array}$ & Privada \\
\hline Cabrera et al. $2020^{93}$ & Español & $\begin{array}{l}\text { Educación } \\
\text { continuada }\end{array}$ & Transversal & Cirugía Andaluza & $\begin{array}{c}\text { Estrategias pedagógicas } \\
\text { (e-learning) }\end{array}$ & Privada \\
\hline
\end{tabular}


- Indicadores de evaluación comparativa

La distribución de los artículos según el cuartil se encuentra en la figura 4, no obstante, el $36 \%$ de los artículos fueron publicados en revistas sin indexación (ISI/SCOPUS).

B. Indicadores relacionados con los autores

Los autores que ocuparon los dos primeros puestos en nuestro listado participaron respectivamente en 19 y 20 artículos incluidos en la revisión. El autor con mayor influencia, según su número de citaciones e índice $\mathrm{H}$, es el que ocupó el primer lugar, con un índice $\mathrm{H}$ de 31 y un número de citaciones totales de 3825. La mayor razón de publicaciones (publicaciones en IEMC / número total de publicaciones) fue obtenida por el autor número 2 (15,5 \%). En la tabla 3 se presentas los cinco autores colombianos con más publicaciones en esta revisión.

\section{Discusión}

De acuerdo con el objetivo del estudio, se considera como primer hallazgo de nuestra investigación que la mayoría de los estudios incluidos fueron escritos en español, con diseño transversal, publicados en revistas colombianas y se enfocaron principalmente en postgrado, tratando en especial temas sobre bienestar y enseñanza clínica. En segundo lugar, en Colombia existe una tendencia creciente en el número de publi-

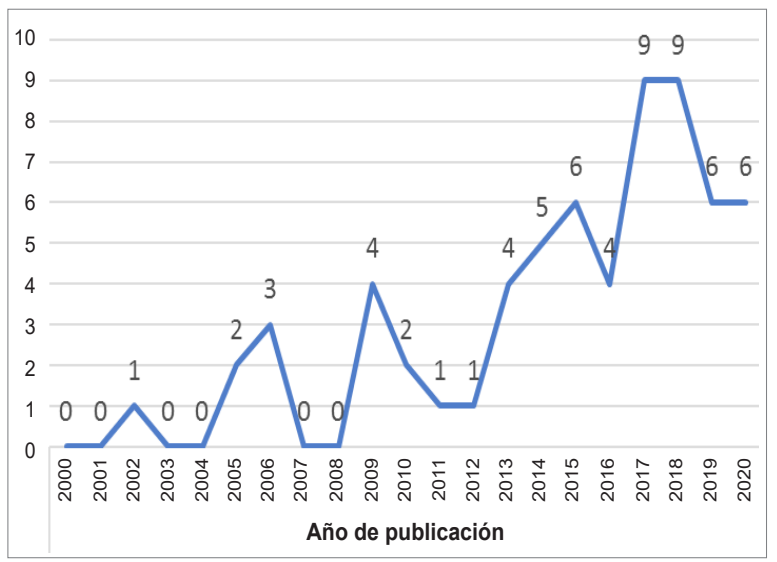

Figura 3. Número de publicación según el año. caciones sobre IEMC a partir de 2013, pero con un bajo número de publicaciones y citaciones en revistas de alto impacto (Q1). Finalmente, la IEMC en Colombia es realizada por un reducido número de autores, en quienes se concentran el mayor número de citaciones e indicadores de influencia, pero solo un $15 \%$ de la producción intelectual de estos autores se relaciona con educación médica.

El primer hallazgo puede explicarse por la necesidad de los cirujanos de evaluar el bienestar de estudiantes y residentes frente a los desafíos de los sistemas educativo y sanitario, con el objetivo de promover mejores condiciones educativas y aspectos relacionados con procedimientos de enseñanza clínica. Este hallazgo es similar al de otros contextos, como el norteamericano, en el que es creciente la evaluación sobre la deserción, el desgaste profesional y el acoso laboral en residentes de cirugía ${ }^{11,30-32}$.

En la presente RB, los estudios sobre bienestar (17,5\% de los artículos seleccionados) evalúan los factores que fomentan la deserción, el desgaste profesional o "burnout" y la carga académica de los estudiantes ${ }^{33-35}$. No obstante, existe una deuda de los investigadores colombianos con la evaluación de otros desenlaces, como el acoso laboral en los programas de residencia, así como los efectos y consecuencias de este problema en el bienestar de los residentes.

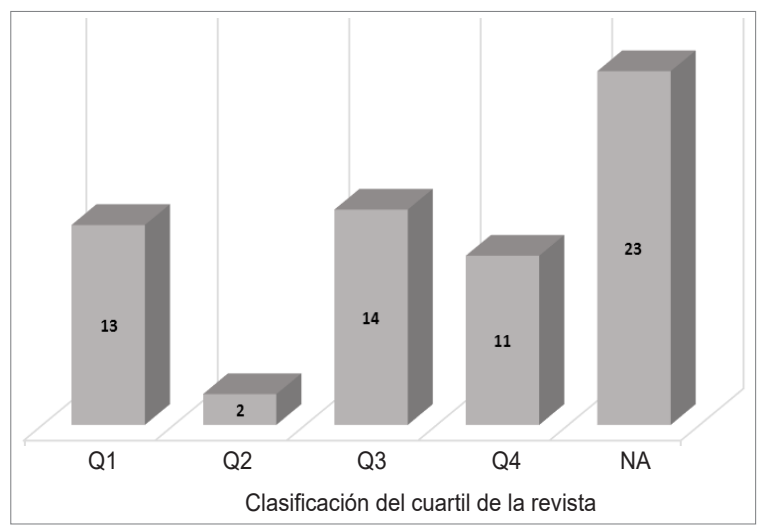

* Q: cuartil de la revista en el año de publicación; NA: no aplica.

Figura 4. Número de publicaciones según el cuartil de revista indexada en el año de publicación. 
Tabla 3. Indicadores de producción científica del artículo y de la revista donde fue publicado.

\begin{tabular}{|c|c|c|c|c|c|}
\hline Referencia & $\begin{array}{l}\text { Número de } \\
\text { Citaciones }\end{array}$ & Revista donde fue publicado & $\begin{array}{l}\text { Índice } \\
\text { H de la } \\
\text { revista }\end{array}$ & $\begin{array}{l}\text { Clasificación } \\
\text { por cuartil de la } \\
\text { revista (año de } \\
\text { publicación) }\end{array}$ & $\begin{array}{l}\text { SJR de la } \\
\text { revista } \\
\text { (año de } \\
\text { publicación) }\end{array}$ \\
\hline Sanabria et al. $2002^{40}$ & 7 & Revista Colombiana de Cirugía & NA & NA & NA \\
\hline Riveros et al. $2005^{41}$ & 3 & Stud Health Technol Inform & 56 & Q4 & 0,202 \\
\hline Villaveces et al. $2005^{42}$ & 24 & Injury Prevention & 78 & Q1 & 1,052 \\
\hline Bermúdez et al. $2006^{43}$ & 27 & Revista Colombiana de Cirugía & NA & NA & NA \\
\hline Isaza et al. $2006^{44}$ & 14 & Revista Ciencias de la Salud & 6 & NA & NA \\
\hline Isaza et al. $2006^{45}$ & 4 & Revista Ciencias de la Salud & 6 & NA & NA \\
\hline Torregrosa et al. $2009^{46}$ & 8 & Revista Colombiana de Cirugía & NA & NA & NA \\
\hline Ruiz-Parra et al. 200929 & 59 & Revista de la Facultad de Medicina & 7 & Q4 & 0,101 \\
\hline Salinas et al. $2009^{37}$ & 21 & Revista Chilena de Ingeniería & NA & NA & NA \\
\hline Bejarano et al. $2009^{47}$ & 7 & Revista Colombiana de Cirugía & NA & NA & NA \\
\hline Tapias et al. $2010^{48}$ & 33 & Revista Colombiana de Cirugía & NA & NA & NA \\
\hline Rubiano et al. $2010^{49}$ & 13 & Prehospital Emergency Care & 61 & Q1 & 1,569 \\
\hline Rojas et al. $2011^{50}$ & 10 & Stud Health Technol Inform & 56 & Q3 & 0,273 \\
\hline Hull et al. $2012^{51}$ & 15 & International Journal of Surgery & 53 & Q2 & 0,562 \\
\hline Henao et al. 201352 & 5 & Biomédica & 26 & Q3 & 0,298 \\
\hline Domínguez et al. 201353 & 5 & Revista Colombiana de Cirugía & NA & NA & NA \\
\hline Sanabria et al. $2013^{54}$ & 2 & Revista Colombiana de Cirugía & NA & NA & NA \\
\hline Amaya et al. $2013^{55}$ & 7 & Revista Colombiana de Cirugía & NA & NA & NA \\
\hline Ramírez et al. $2014{ }^{56}$ & 6 & Revista Ciencias de la Salud & 5 & Q4 & 0,108 \\
\hline Bustamante et al. $2014^{57}$ & 10 & Biomédica & 26 & Q3 & 0,290 \\
\hline Bustamante et al. $2014{ }^{58}$ & 1 & Revista Colombiana de Cirugía & NA & NA & NA \\
\hline Amaya et al. $2014{ }^{59}$ & 21 & International Journal of Surgery & 53 & Q2 & 0,552 \\
\hline Quintero et al. $2014{ }^{60}$ & 56 & BMC Medical Education & 61 & Q1 & 0,843 \\
\hline Galindo et al. $2015^{24}$ & 3 & latreia & 9 & Q4 & 0,116 \\
\hline Amaya et al. $2015^{61}$ & 17 & Revista Colombiana de Anestesiología & 0 & Q3 & 0,159 \\
\hline Mantilla et al. $2015^{33}$ & 3 & Universitas Medica & NA & NA & NA \\
\hline Olmos-Vega et al. $2015^{62}$ & 19 & BMC Medical Education & 61 & Q1 & 0,757 \\
\hline Domínguez et al. $2015^{39}$ & 44 & Biomédica & 26 & Q3 & 0,317 \\
\hline Domínguez et al. $2015^{63}$ & 1 & Medical Education & 129 & Q1 & 2,085 \\
\hline Barrera et al. $2016^{64}$ & 1 & Revista Ciencias de la Salud & 5 & Q4 & 0,138 \\
\hline González-Neira et al. $2016^{65}$ & 0 & Colombia Medica & 16 & Q3 & 0,375 \\
\hline Isaza et al. $2016^{34}$ & 13 & Revista Ciencias de la Salud & 6 & Q4 & 0,138 \\
\hline Quintero et al. $2016^{66}$ & 31 & $\begin{array}{c}\text { Journal of Medical Education and Curricular } \\
\text { Development }\end{array}$ & NA & NA & NA \\
\hline Bayter et al. $2017^{67}$ & 5 & Repertorio de Medicina y Cirugía. & NA & NA & NA \\
\hline Olmos et al. $2017{ }^{68}$ & 27 & Medical Education & 129 & Q1 & 2,036 \\
\hline Cervera et al. $2017^{69}$ & 0 & Investigación en Educación Médica & NA & NA & NA \\
\hline Domínguez et al. $2017^{70}$ & 3 & Revista Colombiana de Cirugía & NA & NA & NA \\
\hline Domínguez et al. $2017^{71}$ & 8 & Revista Colombiana de Anestesiología & 0 & Q3 & 0,154 \\
\hline
\end{tabular}


Continuación tabla 3

\begin{tabular}{|c|c|c|c|c|c|}
\hline Referencia & $\begin{array}{l}\text { Número de } \\
\text { Citaciones }\end{array}$ & Revista donde fue publicado & $\begin{array}{l}\text { Índice } \\
\text { H de la } \\
\text { revista }\end{array}$ & $\begin{array}{l}\text { Clasificación } \\
\text { por cuartil de la } \\
\text { revista (año de } \\
\text { publicación) }\end{array}$ & $\begin{array}{l}\text { SJR de la } \\
\text { revista } \\
\text { (año de } \\
\text { publicación) }\end{array}$ \\
\hline Domínguez et al. $2017^{72}$ & 8 & Educación Medica & 12 & Q3 & 0,236 \\
\hline Domínguez et al. $2017^{73}$ & 1 & Educación Medica & 12 & Q3 & 0,236 \\
\hline Ramírez et al. $20177^{35}$ & 0 & latreia & 9 & Q4 & 0,117 \\
\hline Herrera-Almario et al. $2017^{36}$ & 20 & The American Journal of Surgery & 140 & Q1 & 1,141 \\
\hline Domínguez et al. $2018^{74}$ & 0 & Educación médica & 12 & Q3 & 0,202 \\
\hline Domínguez et al. $2018^{75}$ & 2 & Revista Chilena de Cirugía & 9 & Q4 & 0,106 \\
\hline Domínguez et al. $2018^{76}$ & 2 & Educación Medica & 12 & Q3 & 0,202 \\
\hline Domínguez et al. $2018^{77}$ & 10 & PLoS ONE & 268 & Q1 & 1,1 \\
\hline Vergel et al. $2018^{78}$ & 3 & Medical Education Online & 24 & Q1 & 0,806 \\
\hline Domínguez et al. $2018^{79}$ & 1 & Educación Medica & 9 & Q3 & 0,202 \\
\hline Sierra et al. $2018^{80}$ & 0 & Revista Colombiana de Cirugía & NA & NA & NA \\
\hline Domínguez et al. $2018^{81}$ & 0 & Educación Médica & 12 & Q3 & 0,202 \\
\hline Moros et al. 2018 (82) & 0 & Revista Chilena de Cirugía & 9 & Q4 & 0,107 \\
\hline Castañeda et al. $2019^{83}$ & 0 & latreia & 9 & Q4 & 0,115 \\
\hline Dominguez et al. $2019^{84}$ & 0 & International Journal of Medical Education & 16 & NA & NA \\
\hline Cortés et al. $2019^{85}$ & 0 & Universitas Medica & NA & NA & NA \\
\hline Dominguez et al. $2019{ }^{86}$ & 1 & Journal of Surgical Research & 104 & Q1 & 0,839 \\
\hline Dominguez et al. $2019^{87}$ & 0 & Biomédica & 26 & Q3 & 0,284 \\
\hline Angarita et al. $2019{ }^{88}$ & 2 & Breast Cancer Research and Treatment & 148 & Q1 & 1,899 \\
\hline Orlas et al. 202089 & 0 & Journal of Surgical Research & 104 & Q1 & 0,839 \\
\hline Dominguez et al. 202090 & 0 & Revista Chilena de Cirugía & 9 & Q4 & 0,106 \\
\hline Cabrera et al. $2020^{91}$ & 1 & Revista Colombiana de Cirugía & NA & NA & NA \\
\hline Sánchez et al. $2020^{38}$ & 0 & Revista Colombiana de Cirugía & NA & NA & NA \\
\hline Cabrera et al. $2020^{92}$ & 0 & Surgery & 148 & Q1 & 1,521 \\
\hline Cabrera et al. $2020^{93}$ & 2 & Cirugía Andaluza & NA & NA & NA \\
\hline
\end{tabular}

*SRJ: Scimago Journal Ranking en relación con el año de publicación del artículo; NA: no aplica; Q: cuartil.

Tabla 3. Indicadores bibliométricos del Autor.

\begin{tabular}{ccccccc}
\hline Autores & $\begin{array}{c}\text { Número de } \\
\text { publicaciones } \\
\text { totales }\end{array}$ & $\begin{array}{c}\text { Número de } \\
\text { publicaciones en } \\
\text { IEMC en Colombia }\end{array}$ & $\begin{array}{c}\text { Número de } \\
\text { citaciones } \\
\text { totales }\end{array}$ & $\begin{array}{c}\text { Índice H } \\
\text { Google }\end{array}$ & $\begin{array}{c}\text { Índice H } \\
\text { SCOPUS }\end{array}$ & $\begin{array}{c}\text { Razón de publicaciones en } \\
\text { IEMC con respecto al número } \\
\text { de publicaciones generales } \\
\text { por autor }\end{array}$ \\
\hline 1 & 246 & 19 & 3825 & 31 & 21 & 7,72 \\
2 & 129 & 20 & 1095 & 17 & 9 & 15,50 \\
3 & 95 & 3 & 570 & 11 & 6 & 3,15 \\
4 & 74 & 7 & 467 & 12 & 1 & 9,54 \\
5 & 70 & 4 & 450 & 8 & 4 & 5,71 \\
\hline
\end{tabular}

*IEMC: Investigación en educación médica en cirugía 
Identificamos que un $12,7 \%$ de las publicaciones realizadas en el país versan sobre simulación clínica. Esta tendencia también es creciente en otros contextos, en donde la restricción del número de horas de práctica ha fortalecido el desarrollo de la simulación, como estrategia para compensar la disminución de oportunidades reales de practica para estudiantes y residentes ${ }^{6-8,36}$. Igualmente, este fenómeno es una respuesta natural al desarrollo de nuevas tecnologías para la enseñanza clínica (e-learning, realidad virtual y aula invertida, entre otros) ${ }^{29,37-39}$.

Los hallazgos bibliométricos tienen algunas explicaciones. Creemos que existe una tendencia creciente en el número de publicaciones por el interés de los autores colombianos en documentar sus experiencias educativas y comunicarlas, y así mismo, crear y utilizar nuevas herramientas de aprendizaje ${ }^{39}$. Esta tendencia puede explicarse por el auge sostenido de la IEMC a nivel global y por la consolidación de la educación quirúrgica como una disciplina con un cuerpo propio de conocimiento. En el medio colombiano existe una concentración de las publicaciones en revistas sin indexación internacional, lo cual podría explicar su bajo número de citaciones, mostrando una necesidad de mejorar la calidad y el alcance de la IEMC. Es limitado el número de estudios que evalúan el estado de la investigación en educación en cirugía en otros países, para realizar comparaciones al respecto.

Este estudio bibliométrico es el primero que se realiza para evaluar el estado de la IEMC mediante un proceso metodológico riguroso, que permite una adecuada identificación, evaluación y síntesis de los resultados obtenidos. Se logró identificar las publicaciones sobre IEMC y, de manera indirecta, determinar un nivel de calidad de estas, acorde con las publicaciones en revistas de alto impacto (Q1 -Q2) que, a la luz de lo anterior, constituye un reto en el mejoramiento de la calidad de la IEMC, en cuanto a equipos de trabajo, metodología de investigación, tema y alcance de las mismas

La principal limitación de este estudio radica en la falta de inclusión y evaluación de otras fuentes de información, como resúmenes de con- greso, tesis de grado y literatura gris, dado que no facilitan el estudio bibliométrico al no tener establecido su indicador de impacto e influencia. No obstante, la producción intelectual documentada en estas fuentes de información es útil para generar nuevos estudios.

Con esta investigación pretendemos motivar a los cirujanos colombianos, a desarrollar investigaciones que contribuyan a fortalecer la producción intelectual nacional sobre educación médica en cirugía. Creemos que es importante generar estudios interinstitucionales y multidisciplinarios para fortalecer este campo del quehacer del cirujano y documentar la experiencia educativa. La aplicabilidad e implementación de los cambios derivados de estas investigaciones permitirán transformar de manera positiva los estudiantes de postgrados y los cirujanos, redundando en un eventual impacto positivo en el desarrollo de su profesión. Asimismo, proponemos que se realicen investigaciones en temas poco explorados como el desarrollo profesional continuo, que abriría una puerta para promover la excelencia académica, mediante la actualización de conocimientos y destrezas para los profesionales especialistas en cirugía general.

\section{Conclusión}

Este estudio identifica el estado de la IEMC en Colombia durante el siglo XXI, sus tendencias e indicadores bibliométricos. Estos resultados proponen caminos para la generación de nuevos estudios, para mejorar la calidad de la producción científica y para promover una mayor influencia de la IEMC, representado en publicaciones en revistas de mayor impacto.

\section{Cumplimiento de normas éticas}

Consentimiento informado: Este estudio no incluyó pacientes, por lo que no se requiere diligenciamiento de consentimiento informado.

Conflicto de interés: Los autores declaran no tener ningún conflicto.

Fuentes de financiación: Este estudio fue financiado con recursos propios. 


\section{Contribución de los autores:}

- Concepción y diseño del estudio: José Manuel SánchezJaramillo, Luis Carlos Domínguez, Neil Valentín Vega, Paula Meneses.

- Adquisición de datos: José Manuel Sánchez-Jaramillo, Luis Carlos Domínguez, Neil Valentín Vega, Paula Meneses.

- Análisis e interpretación de datos: José Manuel SánchezJaramillo, Luis Carlos Domínguez, Neil Valentín Vega, Paula Meneses.

- Redacción del manuscrito: José Manuel SánchezJaramillo, Luis Carlos Domínguez, Neil Valentín Vega, Paula Meneses.

- Revisión crítica: José Manuel Sánchez-Jaramillo, Luis Carlos Domínguez, Neil Valentín Vega, Paula Meneses.

\section{Referencias}

1. Mcllhenny C, Kurashima Y, Chan C, Hirano S, Domínguez-Rosado I, Stefanidis D. General surgery education across three continents. Am J Surg. 2018;215:209-13. https://doi.org/10.1016/j.amjsurg.2017.12.002

2. Patel M. Changes to postgraduate medical education in the 21st century. Clin Med (Lond.). 2016;16:311-4. https://doi.org/10.7861/clinmedicine.16-4-311

3. Ferguson PC, Caverzagie KJ, Nousiainen MT, Snell L, ICBME Collaborators. Changing the culture of medical training: An important step toward the implementation of competency-based medical education. Med Teach. 2017;39:599-602. https://doi.org/10.1080/0142159X.2017.1315079

4. Lombardi BMM. Authentic learning for the 21st century: An overview. Oblinger DG, ed. Learning. 2007. Fecha de consulta:11 de enero de 2020. Disponible en: https://www.researchgate.net/publication/220040581

5. McCoy L, Lewis JH, Dalton D. Gamification and multimedia for medical education: A landscape review. J Am Osteopath Assoc. 2016;116:22-34. https://doi.org/10.7556/jaoa.2016.003

6. Cheston CC, Flickinger TE, Chisolm MS. Social media use in medical education: A systematic review. Acad Med. 2013;88:893-901.

https://doi.org/10.1097/ACM.0b013e31828ffc23

7. Kaiser LR, Mullen JL. Surgical education in the new millennium: The university perspective. Surg Clin North Am. 2004;84:1425-39. https://doi.org/10.1016/j.suc.2004.06.012

8. Dietl CA, Russell JC. Effects of technological advances in surgical education on quantitative outcomes from residency programs. J Surg Educ. 2016;73:819-30. https://doi.org/10.1016/j.jsurg.2016.03.016

9. Brunckhorst O, Khan MS, Dasgupta P, Ahmed K. Nontechnical skill training and the use of scenarios in modern surgical education. Curr Opin Urol. 2017;27:330-6. https://doi.org/10.1097/MOU.0000000000000402
10. Hoffman RL, Morris JB, Kelz RR. Surgical residency training at a university-based academic medical center. Surg Clin North Am. 2016;96:59-70. https://doi.org/10.1016/j.suc.2015.09.004

11. Khoushhal Z, Hussain MA, Greco E, Mamdani M, Verma $\mathrm{S}$, Rotstein $\mathrm{O}$, et al. Prevalence and causes of attrition among surgical residents. A systematic review and meta-analysis. JAMA Surg. 2017;152:265-72. https://doi.org/10.1001/jamasurg.2016.4086

12. Tavakol M, Sandars J. Quantitative and qualitative methods in medical education research: AMEE Guide No 90: Part I. Med Teach. 2014;36:746-56. https://doi.org/10.3109/0142159X.2014.915298

13. Azer SA, Dupras DM, Azer S. Writing for publication in medical education in high impact journals. Eur Rev Med Pharmacol Sci. 2014;18:2966-81.

14. Leon LG. El cirujano y la escritura: algunas reflexiones. Cirujano General. 2001;23:54-7.

15. Thompson DF, Walker CK. A descriptive and historical review of bibliometrics with applications to medical sciences. Pharmacotherapy. 2015;35:551-9. https://doi.org/10.1002/phar.1586

16. Thomson Reuters. Whitepaper using bibliometric: A guide to evaluating research performance with citation data. 2008. Fecha de consulta: 11 de junio de 2020. Disponible en: https://services.anu.edu.au/files/system/ Pendlebury_White_Paper.pdf

17. Escorcia TA. El análisis bibliométrico como herramienta para el seguimiento de publicaciones científicas, tesis y trabajos de grado. Pontificia Universidad Javeriana. Director. 2008;1-61. Fecha de consulta: 16 de julio de 2020. Disponible en: https://repository.javeriana.edu. co/bitstream/handle/10554/8212/tesis209.pdf?sequence $=1$

18. Sharma B, Boet S, Grantcharov T, Shin E, Barrowman NJ, Bould MD. The h-index outperforms other bibliometrics in the assessment of research performance in general surgery: A province-wide study. Surgery. 2013;153:493-501. https://doi.org/10.1016/j.surg.2012.09.006

19. Turaga KK, Gamblin TC. Measuring the surgical academic output of an institution: The "institutional" H-index. J Surg Educ. 2012;69:499-503. https://doi.org/10.1016/j.jsurg.2012.02.004

20. Heldwein FL, Hartmann AA, Kalil AN, Neves BVD, Ratti GSB, Beber MC, et al. Cited brazilian papers in general surgery between 1970 and 2009. Clinics. 2010;65:521-9. https://doi.org/10.1590/S1807-59322010000500010

21. Azer SA. The top-cited articles in medical education: A bibliometric analysis. Acad Med. 2015;90:1147-61. https://doi.org/10.1097/ACM.0000000000000780

22. Gutiérrez VP. Surgical education in Argentina. World J Surg. 2010;34:877-9. https://doi.org/10.1007/s00268-010-0501-9 
23. Braghetto I. Surgical training in Chile. World J Surg. 2010;34:884-6. https://doi.org/10.1007/s00268-010-0503-7

24. Galindo-Cárdenas LA, López-Núñez JA, Arango-Rave ME, Vallejo-Merino I. Tendencias de la investigación sobre educación en los posgrados médicos. Iatreia. 2015;28:434-42.

25. Urrútia G, Bonfill X. Declaración PRISMA: una propuesta para mejorar la publicación de revisiones sistemáticas y metaanálisis. Med Clin. 2010;135:507-11. https://doi.org/10.1016/j.medcli.2010.01.015

26. van Eck NJ, Waltman L. Software survey: VOSviewer, a computer program for bibliometric mapping. Scientometrics. 2010;84:523-38.

https://doi.org/10.1007/s11192-009-0146-3

27. Higgins JPT, Thomas J, Chandler J, Cumpston M, Li T, Page MJ, Welch VA (editors). Cochrane Handbook for Systematic Reviews of Interventions Version 5.1.0. The EQUATOR Network. Fecha de consulta: 11 de junio de 2020. Disponible en: https://www.equator-network. org/reporting-guidelines/cochrane-handbook-for-systematic-reviews-of-interventions-version-5-1-0/

28. Torres-Salinas D, Ruiz-Pérez R, Delgado-López-Cózar E. Google Scholar como herramienta para la evaluación científica. El profesional de la información. 2009;18:501-10. https://doi.org/10.3145/epi.2009.sep.03

29. Ruiz-Parra AI, Angel-Müller E, Guevara O. La simulación clínica y el aprendizaje virtual. Tecnologías complementarias para la educación médica. Rev Fac Med. 2009;57:67-79.

30. Zhang LM, Ellis RJ, Ma M, Cheung EO, Hoyt DB, Bilimoria KY, Hu YY. Prevalence, types, and sources of bullying reported by US general surgery residents in 2019. JAMA. 2020;323:2093-5. https://doi.org/10.1001/jama.2020.2901

31. Gifford E, Galante J, Kaji AH, Nguyen V, Nelson MT, Sidwell RA, et al. Factors associated with general surgery residents' desire to leave residency programs: A multi-institutional study. JAMA Surg. 2014;149:948-53. https://doi.org/10.1001/jamasurg.2014.935

32. Fitzgerald CA, Smith RN, Luo-Owen X, Turay D, Ferrada $\mathrm{P}$, Han J, et al. Screening for harassment, abuse, and discrimination among surgery residents: An EAST multicenter trial. Am Surg. 2019;85:456-61.

33. Mantilla-Flórez J, Suárez A, Laverde A, Moros JG, González-Neira EM, Suárez DR. Efecto del entrenamiento mental en la capacitación de aprendices en cirugía laparoscópica. Univ Méd. 2015;56:400-11

34. Isaza-Restrepo A, Enríquez-Guerrero C, Pérez-Olmos I. Deserción y rezago académico en el programa de medicina de la Universidad del Rosario, Bogotá, Colombia. Rev Cienc Salud. 2016;14:231-45. http://dx.doi.org/10.12804/revsalud14.02.2016.08

35. Ramírez-Sanchez NA, Vega-Peña NV, Domínguez-Torres LC, Sanabria-Quiroga ÁE. El entrenamiento mental y los cirujanos: una estrategia de mejoramiento. Iatreia. 2018;31:180-90.

http://dx.doi.org/10.17533/udea.iatreia.v31n2a06

36. Herrera-Almario GE, Kirk K, Guerrero VT, Jeong K, Kim $\mathrm{S}$, Hamad GG. The effect of video review of resident laparoscopic surgical skills measured by self- and external assessment. Am J Surg. 2016;211:315-20.

https://doi.org/10.1016/j.amjsurg.2015.05.039

37. Salinas SA, Vivas Albán OA. Modelado, simulación y control del robot para cirugía laparoscópica "Lapbot." Ingeniare. Rev Chil Ing. 2009;17:317-28.

http://dx.doi.org/10.4067/S0718-33052009000300005

38. Sánchez-Ussa S, Ariza-Gutiérrez A. Educación quirúrgica en Colombia en la era del COVID-19. Rev Colomb Cir. 2020;35:251-6. https://doi.org/10.30944/20117582.631

39. Domínguez LC, Vega NV, Espitia EL, Sanabria ÁE, Corso C, Serna AM, Osorio C. Impacto de la estrategia de aula invertida en el ambiente de aprendizaje en cirugía: Una comparación con la clase magistral. Biomedica. 2015;35:513-21. https://doi.org/10.7705/biomedica.v35i4.2640

40. Sanabria A, Jaime-Contreras M, Aristizabal H. Experiencia quirurgica de la seccion cirugia general de la Universidad de Antioquia Hospital San Vicente de Paul. Rev Colomb Cir. 2002;17:199-206.

41. Riveros RE, Espinosa A, Jimenez P, Martinez L. E-learning experience: A teaching model with undergraduate surgery students in a developing country. Stud Health Technol Inform. 2005;111:404-6.

42. Villaveces A, Kammeyer JA, Bencevic H. Injury prevention education in medical schools: an international survey of medical students. Inj Prev. 2005;11:343-7. https://doi.org/10.1136/ip.2005.009118

43. Bermúdez CE, Monro- Melo A, Torregrosa L, Henao-Pérez F. Estado actual de la formación de residentes de cirugía general en Colombia. Rev Colomb Cir. 2006;21:225-39.

44. Isaza-Restrepo A, Quiroga-Chand A, Roa-González CN, Delgado-Vega AM, Riveros-Pérez AM. Tutores pares en la facultad de medicina. Rev. Cienc. Salud. 2006;4:12235.

45. Isaza-Restrepo A, Pérez-Olmos I, Martínez LE, Velásquez A, Mercado MA. El tiempo de los estudiantes de medicina en las rotaciones clínicas y el sistema de créditos. Rev Cienc Salud. 2006;4:136-46.

46. Torregrosa L, Tawil M, Ayala JC, Ochoa ÓA, Gil F. Excelencia en la enseñanza en salas de cirugía: cuando los residentes hablan. Rev Colomb Cir. 2009;24:165-74.

47. Bejarano M. Producción intelectual de las universidades y de los hospitales docentes en Colombia. Rev Colomb Cir. 2009;24:14-5.

48. Tapias-Vargas LF, Torres SA, Tapias-Vargas L, Santamaría CM, Valencia-Ángel LI, Orozco-Vargas LC. Accidentes biológicos en médicos residentes de Bucaramanga, Colombia. Rev Colomb Cir. 2010;25:290-99. 
49. Rubiano AM, Sánchez ÁI, Guyette F, Puyana JC. Trauma care training for National Police nurses in Colombia. Prehospital Emerg Care. 2010;14:124-30. https://doi.org/10.3109/10903120903349762

50. Rojas D, Cristancho S, Rueda C, Grierson L, Monclou A, Dubrowski A. The validation of an instrumented simulator for the assessment of performance and outcome of knot tying skill: A pilot study. Stud Health Technol Inform. 2011;163:517-23.

51. Hull L, Arora S, Amaya AC, Wheelock A, Gaitán-Duarte $\mathrm{H}$, Vincent C, Sevdalis N. Building global capacity for patient safety: A training program for surgical safety research in developing and transitional countries. Int J Surg. 2012;10:493-9. https://doi.org/10.1016/j.ijsu.2012.07.005

52. Henao O, Escallón J, Green J, Farcas M, Sierra JM, Sánchez W, Okrainec A. Fundamentos de cirugía laparoscópica en Colombia con telesimulación: una herramienta adicional para la formación integral de cirujanos. Biomédica. 2013;33:107-14. http://doi.org/10.7705/biomedica.v33i1.1425

53. Domínguez LC, Sanabria ÁE, Vega NV, Espitia EL, Cabrera EY, Osorio C. Factores que influencian la elección de una carrera quirúrgica: análisis multiinstitucional global desde una perspectiva académica en Bogotá. Rev Colomb Cir. 2013;28:275-81.

54. Sanabria ÁE, Vega NV, Domínguez LC, Osorio C, Espitia EL, Serna A. La visita quirúrgica ideal: elementos para su correcta implementación. Rev Colomb Cir. 2013;28:136-44.

55. Amaya AC, Narváez R, Eslava-Schmalbach J. Trabajo en equipo como factor contribuyente en la ocurrencia de errores médicos o eventos adversos. Rev Colomb Cirugía. 2013;28:297-310.

56. Gómez-Ramírez MF, Gómez JC, González-Neira EM, Rúgeles S, Suárez DR, Torregrosa L. Evaluación del mejoramiento de habilidades básicas para cirugía laparoscópica por medio del entrenamiento con un videojuego. Rev Cienc Salud. 2014;12:9-20. http://dx.doi.org/10.12804/10.12804/revsalud12. esp.2014.02

57. Bustamante E, Sanabria Á. Spanish adaptation of the Penn State College of Medicine scale to assess professionalism in medical students. Biomédica. 2014;34:291-9. http://dx.doi.org/10.7705/biomedica.v34i2.1807

58. Bustamante E, Sanabria Á. Evaluación de las actitudes hacia el profesionalismo en estudiantes de medicina. Rev Colomb Cirugía. 2014;29:222-9.

59. Amaya-Arias AC, Barajas R, Eslava-Schmalbach JH, Wheelock A, Gaitán-Duarte H, Hull L, Sevdalis N. Translation, cultural adaptation and content re-validation of the observational teamwork assessment for surgery tool. Int J Surg. 2014;12:1390-402. http://doi.org/10.1016/j.ijsu.2014.10.001

60. Quintero GA. Medical education and the healthcare system - why does the curriculum need to be reformed?
BMC Med. 2014;12:213. https://doi.org/10.1186/s12916-014-0213-3

61. Amaya-Arias AC, Idarraga D, Giraldo V, Gómez LM. Efectividad de un programa para mejorar el trabajo en equipo en salas de cirugía. Rev Colomb Anestesiol. 2015;43:68-75

62. Olmos-Vega F, Dolmans D, Donkers J, Stalmeijer RE. Understanding how residents' preferences for supervisory methods change throughout residency training: A mixed-methods study. BMC Med Educ. 2015;15:177. https://doi.org/10.1186/s12909-015-0462-7

63. Domínguez LC, Espitia EL, Vega NV, Sanabria ÁE. Teamwork attitude: Not just a student issue. Med Educ. 2015;49:1154-5. https://doi.org/10.1111/medu.12872

64. Barrera-Álvarez CA, Salamanca-Santos JD, González-Neira EM, Suárez DR, Rúgeles S. Sistema de evaluación de habilidades psicomotrices en cirujanos de laparoscopia. Revista Ciencias de la Salud. 2016;14:57-67. https://dx.doi.org/10.12804/revsalud14.especial.2016.04

65. González-Neira EM, Jiméne- Mendoza CP, Suarez DR, Rúgeles-Quintero S. Objective evaluation of surgical competency for minimally invasive surgery with a collection of simple tests. Colomb Med. 2016;47:45-50. https://doi.org/10.25100/cm.v47i1.1706

66. Quintero GA, Vergel J, Arredondo M, Ariza MC, Gómez P, Pinzon-Barrios AM. Integrated medical curriculum: advantages and disadvantages. J Med Educ Curric Dev. 2016;3:17-24. https://doi.org/10.4137/JMECD.S18920

67. Bayter E, Cordoba A, Messier J. Residentes como docentes en la Fundación Universitaria de Ciencias de la Salud (FUCS), proyecto de residentes formadores. Repertorio de Medicina y Cirugía. 2017;26:27-34. https://doi.org/10.1016/j.reper.2017.03.001

68. Olmos-Vega FM, Dolmans DHJM, Vargas-Castro N, Stalmeijer RE. Dealing with the tension: how residents seek autonomy and participation in the workplace. Med Educ. 2017;51:699-707. https://doi.org/10.1111/medu.13326

69. Cervera S, Márquez-Ustáriz A, Sánchez-Cortes EF. Evaluación de competencias quirúrgicas en residentes de cirugía general mediante el uso de smartphone. Investig en Educ Médica. 2017;6:130.

70. Domínguez LC, Sanabria Á, Ramírez A, Vargas F, Pacheco M, Jiménez G. Desgaste profesional en residentes colombianos de cirugía: resultados de un estudio nacional. Rev Colomb Cir. 2017;32:121-7. http://dx.doi.org/10.30944/20117582.16

71. Domínguez LC, Sierra D, Pepín JJ, Moros G, Villarraga A. Efecto del Aula Invertida Extendida a simulación clínica para la resucitación del paciente traumatizado: Estudio piloto de las percepciones estudiantiles sobre el aprendizaje. Rev Colomb Anestesiol. 2017;45:4-11.

72. Dominguez LC, Sanabria ÁE, Sierra DO. El clima productivo en cirugía: ¿una condición para el aprendizaje 
en el aula invertida? Educ Med. 2018;19:263-9. https://doi.org/10.1016/j.edumed.2017.08.001

73. Domínguez LC, Urueña N, Sanabria Á, Pepín JJ, Mosquera M, Vega NV, Osorio C. Autonomía y supervisión del residente de cirugía. ¿Se cumplen las expectativas en el quirófano? Educ Med. 2018;19:208-16.

74. Domínguez LC, Sanabria AE. Validez de constructo y confiabilidad del ROTA-Q para la evaluación de la calidad académica de las rotaciones clínicas en estudiantes de medicina. Educ Med. 2019;20:71-8. https://doi.org/10.1016/j.edumed.2017.11.010

75. Domínguez LC, Sanabria Á, Sierra D. ¿Cómo perciben los estudiantes el clima de aprendizaje en el aula invertida en cirugía?: Lecciones aprendidas y recomendaciones para su implementación. Rev Chil Cir. 2018;70:140-6. http://dx.doi.org/10.4067/s0718-40262018000200140

76. Domínguez LC. Instrumentos para la evaluación del clima de aprendizaje en residencias médicas: síntesis de la evidencia a la luz de las definiciones psicométricas. Educ Med. 2018;19:335-49. https://doi.org/10.1016/j.edumed.2017.10.015

77. Dominguez LC, Stassen L, de Grave W, Sanabria A, Alfonso E, Dolmans D. Taking control: Is job crafting related to the intention to leave surgical training? PLoS One. 2018;13:e0197276. https://doi.org/10.1371/journal.pone.0202293

78. Vergel J, Quintero GA, Isaza-Restrepo A, Ortiz-Fonseca M, Latorre-Santos C, Pardo-Oviedo JM. The influence of different curriculum designs on students' dropout rate: a case study. Med Educ Online. 2018;23:1432963. https://doi.org/10.1080/10872981.2018.1432963

79. Dominguez LC, Vega NV. Efectos del mapa conceptual sobre la síntesis de información en un ambiente de aprendizaje interactivo: Un estudio preexperimental. Educ Med. 2020;21:193-7. https://doi.org/10.1016/j.edumed.2018.08.002

80. Sierra-Sierra S, Sierra-Sierra A, Peñaloza-Barrera C, Uribe-Valencia A, Durán-Meléndez MÁ. Diseño de bitácora centrado en el residente: conociendo y mejorando la experiencia de usuario. Rev Colomb Cir. 2018;33:398405. https://doi.org/10.30944/20117582.87

81. Domínguez LC, Sierra D, Sanabria Á, Restrepo J. ¿Soy parte del equipo?: transformando las percepciones estudiantiles en un instrumento para evaluar el trabajo en equipo en cirugía. Educ Med. 2019;20:213-20. https://doi.org/10.1016/j.edumed.2018.11.005

82. Moros G, Domínguez LC, Sierra D, Villarraga A, Pepín JJ. Actividades profesionales confiables en cirugía mínimamente invasiva: iniciando el camino para lograr competencias en el futuro graduado de medicina. Rev Chil Cir. 2018;70:503-9.

http://dx.doi.org/10.4067/s0718-40262018000600503

83. Castañeda-Motta C, Vega-Peña NV. Reuniones de morbilidad y mortalidad, ¿una estrategia de aprendizaje? Iatreia. 2020;33:286-97.

https://doi.org/10.17533/udea.iatreia.52
84. Dominguez LC, Silkens M, Sanabria A. The Dutch residency educational climate test: construct and concurrent validation in Spanish language. Int J Med Educ. 2019;10:138-48. https://doi.org/10.5116/ijme.5d0c.bff7

85. Cuevas-López L, Cortés-Murgueitio N, Díaz-Castrillon CE, Pinzón F, Molina GR. Experiencia y curva de aprendizaje de apendicectomía y colecistectomía laparoscópica de los residentes de cirugía general en un hospital latinoamericano. Univ Médica. 2019;60:1-10.

https://doi.org/10.11144/Javeriana.umed60-2.curv

86. Dominguez LC, Dolmans D, de Grave W, Sanabria A, Stassen LP. Job crafting to persist in surgical training: A qualitative study from the resident's perspective. J Surg Res. 2019;239:180-90. https://doi.org/10.1016/j.jss.2019.02.005

87. Domínguez LC, Vega V, Sanabria Á, Restrepo J. Construcción de puentes entre la medicina y las humanidades: papel del soporte y la articulación en el aprendizaje en cirugía. Biomedica. 2019;39:55-64. https://doi.org/10.7705/biomedica.v39i1.4012

88. Angarita FA, Price B, Castelo M, Tawil M, Ayala JC, Torregrossa L. Improving the competency of medical students in clinical breast examination through a standardized simulation and multimedia-based curriculum. Breast Cancer Res Treat. 2019;173:439-45. https://doi.org/10.1007/s10549-018-4993-6

89. Orlas CP, Parra MW, Herrera-Escobar JP, Meléndez JJ, Serna JJ, Angamarca E, et al. The challenge of implementing the "Stop the Bleed" campaign in Latin America. J Surg Res. 2020;246:591-8. https://doi.org/10.1016/j.jss.2019.09.042

90. Dominguez-Torres LC, Sierra D, Vega V, Gómez L, Pacheco M, Vargas F. ¿Tienes lo que hay que tener?: Un análisis de los factores asociados con la intención de optar por una carrera en cirugía entre estudiantes de medicina. Rev Cirugia. 2020;72(1). https://doi.org/10.35687/s2452-45492020001451

91. Cabrera-Vargas LF, Luna-Jaspe C, Pedraza-Ciro M. Adaptación de la residencia de Cirugía General en Colombia a la pandemia del COVID-19: programa de enseñanza quirúrgica virtual. Rev Colomb Cir. 2020;35:256-63. https://doi.org/10.30944/20117582.632

92. Cabrera LF, Ferrada P, Mayol J, Mendoza AC, Herrera-Almario G, Pedraza-Ciro M, Sánchez S. Impact of social media on the continuous education of the general surgeon, a new experience, @Cirbosque: A Latin American example. Surgery. 2020;167:890-4. https://doi.org/10.1016/j.surg.2020.03.008

93. Vargas LC, Herrera G, Zuchini AM. Aplicación de las redes sociales en la educación del cirujano general. @ Cirbosque rompiendo el paradigma. Cir Andal. 2020;31:15-20.

https://doi.org/10.37351/2020311.2 